\title{
Tidal volume monitoring by electrical impedance tomography (EIT) using different regions of interest (ROI): Calibration equations
}

\author{
M. Balleza-Ordaz ${ }^{\mathrm{a}}$, E. Perez-Aldaya, M. Vargas-Lunaa, P.J. Riu ${ }^{\mathrm{b}}$ \\ a Guanajuato University, Science and Engineering Department, León, Guanajuato, Mexico \\ b Universitat Politècnica de Catalunya, Electronics and Biomedical Instrumentation Department, Barcelona, Spain
}

Received 26 April 2014

Received in revised form 20 November 2014

Accepted 15 December 2014

Keywords: Electrical impedance tomography (EIT), Respiration Tidal volume, Regions of interest, COPD

Biomedical Signal Processing and Control 18 (2015) 102-109 $\quad$ (c) 2014 Elsevier Ltd. All rights reserved.

http://dx.doi.org/10.1016/j.bspc.2014.12.004

\begin{abstract}
a bstract
A set of calibration equations was previously obtained to transform the lung impedance changes obtained by electrical impedance tomography (EIT), using all frame's elements, into a measurable volume signal. In order to study the goodness of the use of regions of interest (ROI) for lung ventilation monitoring, we considered 6 different ROI to obtain a calibration equation for each area. Our aim was to compare the results, determined by these areas, and those obtained by using all EIT image elements. Two ROI's were defined by those pixels with an impedance change higher than $30 \%$ and $70 \%$ of the maximum change value. These areas were called P30 and P70, respectively. Two other ROI were defined by bounding two areas by mouse, resembling P30 and P70 regions, which were called M30 and M70, respectively. The remainder was defined by two elliptical areas with an eccentricity of 0.8 , and 25 and 32 pixels of mayor axis (E25p and E32p, respectively). Twenty healthy males and 24 chronic obstructive pulmonary disease (COPD) patients were considered. For small region (P30 and M30) we obtained a large dispersion in volume measurement, concluding that small regions are not suitable for monitoring the tidal changes in lung volume even for healthy subjects. The results obtained by the remainder areas, and by using EIT image were similar. Even a slight improvement in data dispersion was obtained by using some ROI. These optimal results, for healthy people, were those corresponding to P70 and M70 (volume dispersion improved from $12 \%$ with the whole EIT image to $9 \%$ using ROI), and for COPD patients improves volume dispersion from $32 \%$ using the whole EIT image to $27 \%$ by using E25p. Using not so small ROI, it is possible to estimate the total lung ventilation.
\end{abstract}

\section{Introduction}

In clinical environment, there is nothing good enough to measure and monitor in a non-invasive manner the breathing pattern (BP), especially during large periods of time. The BP monitoring provides information about neuromuscular alterations suffered by patients and assesses organs that regulate breathing $[1,2]$. The reference method aimed to monitor the BP is the pneumotachometer. However, this device spontaneously modifies the behavior of respiration between $15 \%$ and $35 \%$ due to the essential use of a mouthpiece and clip-nose [3-6]. The pneumotachometer is mainly used in exercise stress testing in order to assess the pulmonary functional capacity during exercise. Other alternative method to monitor BP is the respiratory inductance plethysmography (RIP).

This equipment is used to estimate lung volume by detecting respiratory movements of chest and abdominal wall. This method has been almost completely abandoned due to calibration problems [7-9]. Nowadays, this technique is used to monitor qualitatively respiration in patients with obstructive sleep apnea/hypopnea syndrome. A new experimental method used in clinical environment is the electrical impedance tomography (EIT). EIT is used to image regional impedance distribution in a cross-sectional area of human body [10-12]. By this technique, it is possible to obtain impedance changes [11] that allow to characterize the internal movements produced by a biological body. Different research groups have used the EIT technique in order to describe the behavior of respiratory pattern (RP) $[12,13]$ and detect lung diseases. Some important pneumological applications by using EIT are: (1) the development of an artificial pneumothorax in a controlled experimental setting [14, 15], (2) the quantification of the amount of fluid volume in lungs $[16,17]$ and $(3)$ the determination of regional pressure-volume curves [18-20].

However, the most important EIT application in pneumology area is the regional lung ventilation monitoring as a function of positive end-expiratory pressure (PEEP) in patients in use of mechanical ventilation [21-23]. The effects of regional lung ventilation were studied in patients with acute lung injury (ALI) [24] or in animals [25-28]. Many research groups are studying the relation between the levels of PEEP and the impedance determinations obtained by EIT in order to find out the optimal PEEP. These groups have found out a good correlation between PEEP and EIT determinations. However, the main interest of these researches is not to find out a mathematical model to predict the variable of interest, in this case PEEP.

Our research group decided to convert the impedance changes into a measurable volume signal using a mathematical calibration model. Different factors that strongly depend on each subject must be considered in the obtaining of a calibration equation. These factors are determined by the anthropometric features such as the thoracic shape and 
dimensions and/or the fat layer thickness. All of them can modify the transmission of electrical current through ribcage. This phenomenon was evidenced in previous studies performed by our research group. In those, a set of calibration equations was obtained for being used by EIT device in a group of healthy volunteers [29] (females and males) and chronic obstructive pulmonary disease (COPD) patients [30]. From the obtained results, it was evidenced that the mathematical adjustment of calibration equation for male was better than that obtained for female. Subsequently, the equation for male was assessed in the group of COPD patients causing a large dispersion in volume measurements. Therefore, an alternative mathematical model was obtained in the patients group in order to adjust the equation for male. This model corrects the error caused by any difference (physical or physiological) between healthy volunteers and COPD patients. In these studies, all mathematical models were obtained by a multivariate linear regression of (1) the anthropometric parameters and (2) the proportionality coefficients defined by the impedance and volume signals [29]. In order to estimate the volume changes, based on the EIT impedance measurements, all the elements of EIT image were used [29, 30].

Our main challenge is to find a mathematical model that allows to transform impedance changes from a ROI, into a measurable volume signal with the least possible error. In our case, we proposed a set of calibration equations based on anthropometric parameters. Other mathematical calibration model was proposed by Nebuya et al. This model was based primary on different parameters related to lungs as absolute lung resistivity, lung density, lung weight, etc. [31]. However, these parameters were theoretical. In this work we pretend to obtain a new set of calibration equations defined by different regions of interest (ROI) in a sample of healthy male volunteers and COPD patients. The ROIs are subareas selected from EIT image by three different methods that were designed for this study by our research group. All calibration equations were determined in the same way as in our previous works [29, 30], using a multivariate linear regression to relate the anthropometric parameters and the coefficients of proportionality estimated by the quotient of impedance (from ROI's) and volume (from pneumotachometer) determinations. Afterward, the resulted calibration equations were assessed in independent groups of volunteers (validation groups). Our hypothesis is that by using a reduced area on EIT image, we can improve the mathematical adjustment of calibration equations and therefore reduce the error in the estimation of tidal changes in total lung volume. The main strategy used to achieve our hypothesis is to compare the volume determinations obtained by EIT, using either different ROI's or all pixels of EIT image, with the measurements determined by pneumotachometer used as gold standard.

\section{Materials and methods}

\subsection{Pneumotachometer}

The pneumotachometer used to monitor the respiratory pattern was a Med Graphics preVent ${ }^{\mathrm{TM}}$ Pneumotach from Medical Graphics Corporation (St. Paul, MN, USA). This monitoring device records signals volume versus time.

\subsection{EIT device (TIE4sys)}

The TIE-4sys device is an EIT prototype designed at the Department of Electronic Engineering, Universitat Politècnica de Catalunya, Barcelona, Spain. It uses 16 electrodes and can obtain 17 frames per second. 16 electrodes (Red Dot $2560-3 \mathrm{M}$, London, Ontario, Canada) were placed around the thoracic cage following the protocol described in a previous work [32]. The system was programmed to apply an electrical current of $1 \mathrm{~mA}$ at $48 \mathrm{kHz}$ through any pairs of adjacent electrodes. Differential voltage at the rest of the pairs of adjacent electrodes was recorded for each injection pair, so redundant measurements were obtained, allowing us to calculate the reciprocity error, which is an estimation of the systematic errors and malfunctions present (such as electrodes with poor contact) $[33,34]$.

A reference frame for each subject is constructed by averaging 200 frames corresponding to about $12 \mathrm{~s}$ of tidal breathing. The small changes of thorax shape during respiration are negligible, so we consider a fix model of thorax. Subsequent dynamic images were obtained as a change from this reference by using a weighted back-projection algorithm. This algorithm is aimed to optimize computing time. The software uses a weight matrix that takes into account the spatial sensitivity of each image's pixel [35]. Once about $30 \mathrm{~s}$ of tidal breathing are recorded and images are reconstructed, a signal that depicts the changes in impedance for every pixel in image over time is constructed. This signal is filtered with a median filter (window width of 5 samples) to avoid artifacts and with a Butterworth filter (order 1; type: low-pass; $\mathrm{fc}=0.8 \mathrm{~Hz}$ ) to avoid the cardiac-related changes [36]. Images are then reassembled.

\subsection{Determination of ROI's}

We defined a set of reduced ROI's by 3 different methods. The delimitation of ROI is carried out on an image showing the standard deviations (SD) of impedance changes for each pixel. SD is obtained from the 200 images used to build up the reference frame. The first method (percentage method) is an automatic procedure used to obtain a ROI. It consists on selecting those pixels showing a value of SD larger than a defined percentage of the maximum SD value. Values of $70 \%$ and $30 \%$ were tested. These areas were called $\mathrm{P} 70$ and $\mathrm{P} 30$, respectively.

The second method (mouse method) is a manual procedure used for getting different areas of interest. It consists on selecting by mouse a set of boundary points in the SD EIT image. Subsequently, these points are automatically joined by software, creating a region of interest. This method is defined as a subjective technique and depends, in a large measure, on the criteria of user. In order to assess the goodness of this method, similar areas to the P70 and P30 obtained by the first method were manually selected. The operator uses the P70 and P30 areas to draw by mouse the outline of each one. The resulting regions were called M70 and M30, respectively. 
Then, the impedance changes corresponding to the areas: P70 and M70, and the areas: P30 and M30 were analyzed, respectively. The mouse and percentage methods are compared because in many situations a handmade determination of a clinical ROI is necessary to be performed in situ in order to obtain a better diagnostics. For example, the ventilatory pattern monitoring in those EIT areas produced by the alveolar recruitment in patients in use of mechanical ventilation [37]. Finally, the third method (ellipse method) consists on selecting those pixels determined by two elliptical masks placed in the SD EIT frame. The user can define the position of center, major axis and eccentricity of each ellipse. In this particular case, one ellipse was defined by a major axis of 25 pixels (this area was called E25p) and the other one was determined by a major axis of 32 pixels (this other was called E32p); both with an eccentricity of 0.8 . The ellipse is placed in the regions of SD image that showed larger impedance changes. The idea of this method is to place on EIT image a fixed area with known features without considering the detailed lung shapes (which are not well delimited). However, it is still possible to consider the type of ventilatory pattern corresponding to each lung pathology. The ellipse method is considered an intermediate alternative between SD percentage and mouse method, because the user only define by hand the center, one axis and eccentricity or both axes, the rest is done by software.

\subsection{Subjects}

A first series ( 12 healthy volunteers and 16 COPD patients) was performed to obtain a set of mathematical-equation models to calibrate the EIT for the assessment of lung volume changes. This series was identified as obtaining subgroups. A second series ( 8 healthy volunteers and 8 COPD patients) was performed to validate the estimated, model-based tidal volumes by EIT versus the tidal volumes assessed by the pneumotachometer (validation subgroups). All healthy male volunteers were selected to be non-smokers and with spirometry parameters within the normal range. No other requirements were set. All COPD patients were selected by medical criteria [38], based on all pulmonary function parameters that reflected an obstructive ventilatory pattern (FEV1/FVC $<70 \%$ and

$\mathrm{FVC} \geq 80 \%$ ).

The tests were performed in sitting pose, in a quiet room at sea level with an ambient temperature of $25{ }^{\circ} \mathrm{C}$ and a relative humidity of $60 \%$. The evaluation of both groups was conducted over 4 weeks always during the morning between 9:00 AM and noon. The present study was approved by Ethics committee of Santa Creu I Sant Pau Hospital, Barcelona, Spain. A written informed consent was read and signed by all volunteers. This research was conducted according to the guidelines outlined in the Declaration of Helsinki [39].

\subsection{Procedure}

In this study, we use the pneumotachometer as gold-standard. Therefore, all volume determinations obtained by EIT, using different ROI, are compared with the volume determinations obtained by the gold-standard.

The pneumotachometer and the EIT device (TIE4sys) were connected to each volunteer at the same time. Both devices operated independently of each other with no mutual interference. The breathing patterns of the volunteers at rest were recorded 3 times for periods of $30 \mathrm{~s}$ with a 3-min rest period between measurements.

Each respiratory maneuver was recorded numerically and graphically. The average of the amplitudes of the obtained cycles was taken for analysis. Prior to measure the breathing pattern of each volunteer, we recorded anthropometric data: height, weight, body mass index, skinfold thickness, gender, age, and the chest perimeters at rest and during maximum inspiration and expiration. In this work, we use a mathematical model that allows to transform the impedance changes into a measurable volume signal, the equation is expressed as following:

$$
\mathrm{Z}=\mathrm{A} \times \mathrm{V}
$$

where $\mathrm{Z}$ is the change of impedance obtained by EIT, using the different areas of interest, $\mathrm{V}$ is the volume determinations obtained by the pneumotachometer and $\mathrm{A}$ is a proportionality coefficient. This coefficient is defined in terms of the different anthropometric parameters $(\mathrm{A}=\mathrm{A}$ [weight, height, body mass index, etc.]).

\subsection{Statistical analysis}

All data were expressed as average and standard deviation (SD) values. The impedance value used for the analysis was the total impedance index (TII) estimated by the sum of all the pixel values from the different areas of interest. The calibration mathematical models were obtained by a multivariate linear regression of the anthropometric parameters and the proportionality coefficients $(A)$ corresponding to each volunteer. These coefficients are estimated by the quotient of the tidal volume mean value (from pneumotachometer) and_TII mean value (TII difference during the respiratory process from the ROI under study) registered from each respiratory exercise. In order to compare the relation between the volume determinations obtained by pneumotachometer and EIT device a linear regression was performed. Once the calibration equations were established, predicted tidal volumes were obtained from impedance data. The comparison between the tidal volume determined by the pneumotachometer and the volume obtained by EIT device, were performed by using Student's t-test, due to the fact that the data follows a normal distribution (K-S, $p<0.05)$. The Bland and Altman plot was performed in order to analyze the dispersion of volume differences $[40,41]$. Values of $p<0.05$ were considered to be statistically significant. 


\section{Results}

3.1. Results in healthy male volunteers

The anthropometric values of the obtaining subgroup of healthy males ( $\mathrm{H}: 12)$ were as follows: age: $27 \pm 8$ years, height: $1.79 \pm 0.09 \mathrm{~m}$, weight: $78 \pm 13 \mathrm{~kg}$, body mass index (BMI): $24.2 \pm 3 \mathrm{~kg} / \mathrm{m2}$. The thoracic measurements at the front, side, back and subscapular skinfolds were $19 \pm 6 \mathrm{~mm}, 19 \pm 10 \mathrm{~mm}, 22 \pm 8 \mathrm{~mm}$ and $24 \pm 9 \mathrm{~mm}$, respectively. The chest circumference at rest and at maximum inspiration and expiration were $90 \pm 7 \mathrm{~cm}, 94 \pm 6 \mathrm{~cm}$ and $88 \pm 7 \mathrm{~cm}$, respectively.

The general mathematical model that describes all calibration equations obtained from the multivariate linear regression, using the 6 different ROI's, is depicted by the following expression:

ACalibration $=$ Cte $+\alpha 1 \times$ SS Sk $+\alpha 2 \times$ Weight $+\alpha 3 \times$ BMI $+\alpha 4 \times$ Height $+\alpha 5 \times$ Age

where ACalibration is the proportionality coefficient estimated in terms of the anthropometric parameters; SS Sk is the subscapular skinfold, BMI is the body mass index, and $\alpha$ i corresponds to the calibration equation coefficients that are shown in Table 1. The determination coefficient $\left(R^{2}\right)$ of all these mathematical models was over $90 \%$.

Table 1. Coefficients of calibration equations obtained in the subgroup $\mathrm{H}$ : 12 . All of them were determined by all six ROI's.

\begin{tabular}{|c|c|c|c|c|c|c|c|c|}
\hline \multirow[t]{2}{*}{ Eq. } & \multirow[t]{2}{*}{$\mathrm{ROI}^{3}$} & \multicolumn{7}{|c|}{ Coefficients $\left(\alpha_{t}\right)$} \\
\hline & & Cte & SS_Sk $\alpha_{1}$ & Weight $\alpha_{2}$ & BMI $\alpha_{3}$ & Height $\alpha_{4}$ & Age $\alpha_{5}$ & $R^{2}$ \\
\hline (3) & E32p & 2341 & -22.8 & -11.9 & - & - & - & 0.953 \\
\hline (4) & E25p & 1117 & -9.4 & -5.7 & - & - & - & 0.954 \\
\hline (5) & M70 & 2224 & -23.8 & -11.1 & - & - & - & 0.941 \\
\hline (6) & M30 & 2996 & - & - & -57 & -664 & - & 0.901 \\
\hline (7) & P70 & 2398 & -25.7 & -12 & - & - & - & 0.949 \\
\hline (8) & P30 & 1669 & - & - & -60 & - & 5.4 & 0.920 \\
\hline (9) & Complete EIT image & 2861 & -29.3 & -14.4 & - & - & - & 0.955 \\
\hline
\end{tabular}

E32p and E25p: elliptical masks with major axis of 32 and 25 pixels, respectively; M70 and M30: ROI's obtained by mouse method; P70 and P30: ROI's obtained by percentage method; Cte: constant term of equation; SS Sk: subscapular skinfold; BMI: body mass index; R2: determination coefficient of mathematical models.

${ }^{a}$ All ROI's are explained in Section 2.3.

Subsequently, all calibration equations were applied in the male validation subgroup $\mathrm{H}: 8$. The anthropometric values of this subgroup were as follows: age: $26 \pm 6$ years, height: $1.78 \pm 0.09 \mathrm{~m}$, weight: $75 \pm 11 \mathrm{~kg}$, BMI: $23.8 \pm 3.8 \mathrm{~kg} / \mathrm{m} 2$. The thoracic skinfold measurements at the front, at side, back and subscapular were $19 \pm 4 \mathrm{~mm}, 18 \pm 7 \mathrm{~mm}, 19 \pm 7 \mathrm{~mm}$ and $20 \pm 7 \mathrm{~mm}$, respectively. The chest circumference at rest and at maximum inspiration and expiration were $88 \pm 5 \mathrm{~cm}$, $93 \pm 5 \mathrm{~cm}$ and $86 \pm 5 \mathrm{~cm}$, respectively. The main volume determination obtained by pneumotachometer in this subgroup $(\mathrm{H}: 8)$ as well as those obtained by TIE4sys, using all calibration equations determined by different ROI's, are shown in Table 2. The differences of volume measurements obtained by both monitoring devices (pneumotachometer and TIE4sys) and its respective statistical analysis (Student's t-test) are shown also in Table 2. From the statistical analysis of the results of Table 2, it was evidenced that the best results for healthy males are those with the lowest volume differences with respect to the pneumotachometer (Eqs. (5) and (7)). These mathematical models were determined by M70 and P70 areas, respectively. Nevertheless all the volume differences analyzed are statistically consistent with zero. Also, there are not significant differences between the dispersions of volume differences obtained by the pneumotachometer and those obtained by TIE4sys using each ROI, excepting the M30 area (see Table 5). The differences of volume determinations obtained by the pneumotachometer and TIE4sys, using the equations mentioned above, corresponding to P70 and M70 areas, were analyzed by a Bland-Altman graphic (see Fig. 1). In the case of P70, this volume difference was of $0.008 \pm 0.053$ I with upper and lower limits of 0.115 and -0.098 I, respectively. In the case of $M 70$, the volume difference was $0.014 \pm 0.049$ I with upper and lower limits of 0.112 and -0.083 I, respectively.

The linear regression graphics and its respective equations of the volume determinations obtained by EIT system, using P70 and M70 areas, and by the pneumotachometer are shown in Fig. 2. In both graphs, it is evidenced that the determination coefficients (R2) are greater than 0.8 , showing a good mathematical relation between both variables 


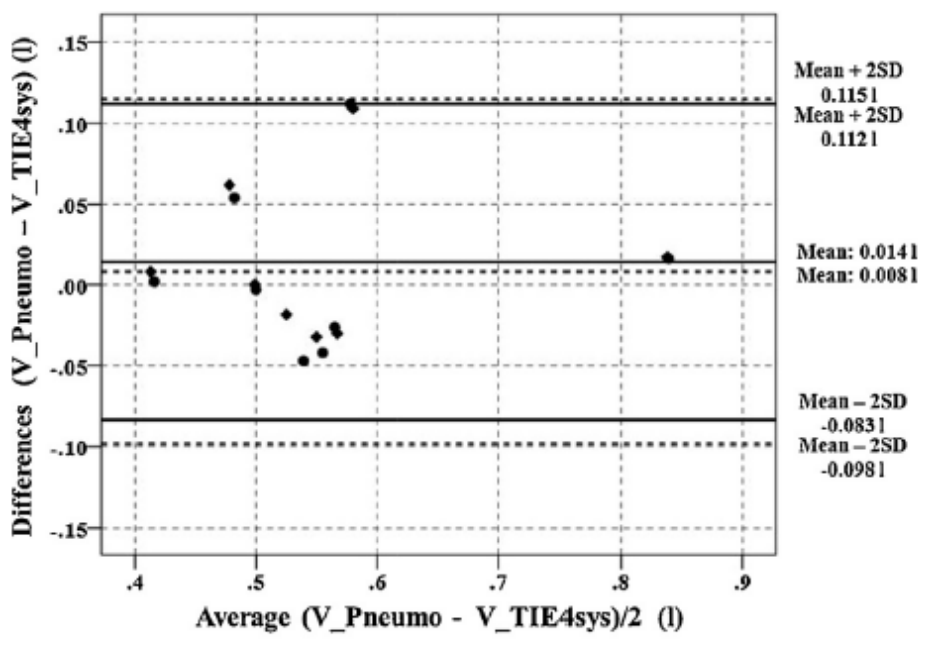

(-) Using equation 4 (M70\%) (--) Using equation 5 (P70\%)

Fig. 1. Bland and Altman graphic. Dispersion of volume differences obtained by pneumotachometer and TIE4sys, using Eq. (4) (ROI: M70\%, rhombus) and Eq. (6) (ROI: P70\%, circles), in the validation male subgroup ( $\mathrm{H}: 8)$.

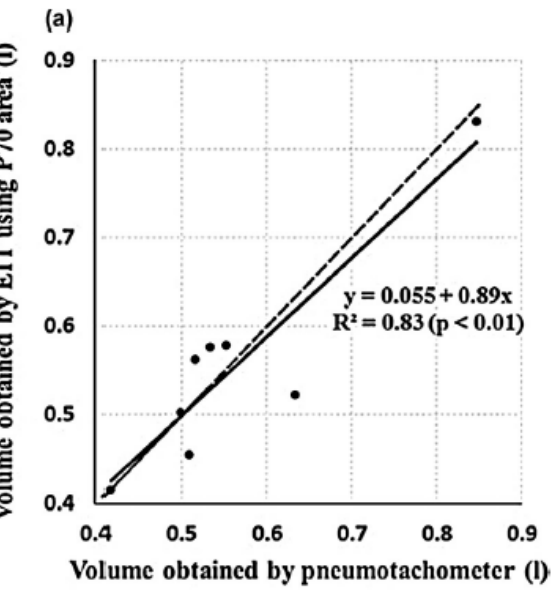

(b)

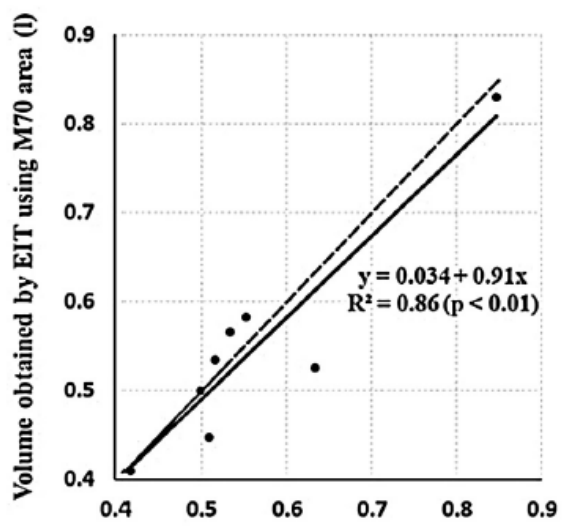

Volume obtained by pneumotachometer (l)

Fig. 2. Linear regression graphic of volume determinations obtained by pneumotachometer and TIE4sys system, using (a) P70 area and (b) M70 area, in the validation group of healthy males $(\mathrm{H}: 8)$. Dashed line represents identical results for both techniques. 
Table 2. Volume determinations obtained by TIE4sys system, using all calibration equations (see Table 1 in the validation male subgroup $(\mathrm{H}: 8)$ ); and the statistical analysis of the differences obtained by both monitoring devices (pneumotachometer and EIT device).

\begin{tabular}{|c|c|c|c|c|c|}
\hline Equation ${ }^{a}(i)$ & ROI $^{\mathrm{f}}$ & Mean estimated volume (1) & Mean volume differences ${ }^{f} \mathrm{Vpn}^{c}-\mathrm{V}(i)^{d}$ & Mean volume dispersion ${ }^{e}$ & $\begin{array}{l}t \text {-Test } \\
p \text {-value }\end{array}$ \\
\hline (3) & E32p & $0.620 \pm 0.149$ & $-0.057 \pm 0.062$ & $11 \%$ & 0.04 \\
\hline (4) & E25p & $0.589 \pm 0.151$ & $-0.026 \pm 0.072$ & $13 \%$ & 0.34 \\
\hline (5) & M70 & $0.549 \pm 0.127$ & $0.014 \pm 0.049$ & $9 \%$ & 0.43 \\
\hline (6) & M30 & $1.462 \pm 2.300$ & $-0.899 \pm 2.268$ & $402 \%$ & 0.30 \\
\hline (7) & P70 & $0.555 \pm 0.126$ & $0.008 \pm 0.053$ & $9 \%$ & 0.67 \\
\hline (8) & P30 & $0.405 \pm 0.478$ & $0.158 \pm 0.495$ & $88 \%$ & 0.39 \\
\hline (9) & $\begin{array}{l}\text { Complete EIT image } \\
\text { Pneumotachometer }^{b}\end{array}$ & $\begin{array}{l}0.574 \pm 0.144 \\
0.564 \pm 0.129\end{array}$ & $-0.010 \pm 0.068$ & $12 \%$ & 0.78 \\
\hline
\end{tabular}

a All equations are shown in Table 1.

b The pneumotachometer is considered in this study as gold standard.

c This value is the volume obtained by pneumotachometer.

d Volume by using calibration equation (i) (see Table 1)

e The volume dispersion was determined respect to the mean of volume measurements obtained by pneumotachometer.

f E32p and E25p: elliptical areas with major axis of 32 and 25 pixels, respectively; M70 and M30: ROI's obtained by mouse method P70 and P30: ROI's obtained by percentage method.

$\mathrm{g}$ The mean estimated volume and the mean volume differences are expressed as mean \pm standard deviation.

Table 3. Coefficients of adjustment equations obtained in the COPD:16 subgroup. All of them were determined by all ROI's.

\begin{tabular}{|c|c|c|c|c|c|c|c|c|}
\hline \multirow[t]{2}{*}{ Eq. } & \multirow[t]{2}{*}{$\mathrm{ROI}^{\mathrm{a}}$} & \multicolumn{7}{|c|}{ Coefficients $\left(\alpha_{t}\right)$} \\
\hline & & Cte & SS_Sk $\beta_{1}$ & Height $\beta_{2}$ & Weight $\beta_{3}$ & Frt_Sk $\beta_{4}$ & Age $\beta_{5}$ & $R^{2}$ \\
\hline (11) & E32p & -5224 & 20.5 & 27,312 & - & - & - & 0.864 \\
\hline (12) & E25p & -2150 & 10.1 & 1048 & - & - & - & 0.880 \\
\hline (13) & M70 & -4387 & 30.2 & 2259 & - & 13.2 & - & 0.912 \\
\hline (14) & M30 & -1158 & - & - & 15 & - & - & 0.910 \\
\hline (15) & $\mathrm{P} 70$ & -6042 & 24.3 & 3152 & - & - & - & 0.916 \\
\hline (16) & P30 & -768 & - & - & 15 & - & -7 & 0.968 \\
\hline (17) & Complete EIT image & -5683 & 27.8 & 2858 & - & - & - & 0.914 \\
\hline
\end{tabular}

E32p and E25p: elliptical masks with major axis of 32 and 25 pixels, respectively; M70 and M30: ROI's obtained by mouse method; P70 and P30: ROI's obtained by percentage method; Cte: constant term of equation; SS Sk and Frt Sk: subscapular and front skinfold; R2: determination coefficient of mathematical models.

${ }^{\text {a } A l l ~ R O I ' s ~ a r e ~ e x p l a i n e d ~ i n ~ S e c t i o n ~ 2.3 . ~}$

Table 4. Volume determinations obtained by TIE4sys, using all adjusted calibration equations for COPD patients, in the validation subgroup of patients (COPD:8); and the statistical analysis of the differences obtained by both monitoring devices (pneumotachometer and EIT device).

\begin{tabular}{|c|c|c|c|c|c|}
\hline Equation $^{\mathrm{a}}(i)$ & ROI $^{f}$ & Mean estimated volume (I)g & Mean volume differencess $\mathrm{Vpn}^{\mathrm{c}}-\mathrm{V}(\mathrm{i})^{d}$ & Mean volume dispersione & $\begin{array}{l}t \text {-Test } \\
p \text {-value }\end{array}$ \\
\hline (3)+(11) & E32p & $0.765 \pm 0.207$ & $-0.016 \pm 0.274$ & $37 \%$ & 0.88 \\
\hline$(4)+(12)$ & E25p & $0.782 \pm 0.198$ & $-0.033 \pm 0.204$ & $27 \%$ & 0.66 \\
\hline$(5)+(13)$ & M70 & $0.787 \pm 0.192$ & $-0.038 \pm 0.273$ & $36 \%$ & 0.70 \\
\hline (6) +(14) & M30 & $0.944 \pm 0.447$ & $-0.195 \pm 0.578$ & $77 \%$ & 0.37 \\
\hline$(7)+(15)$ & P70 & $0.796 \pm 0.247$ & $-0.047 \pm 0.358$ & $48 \%$ & 0.72 \\
\hline$(8)+(16)$ & P30 & $0.235 \pm 1.802$ & $0.513 \pm 1.697$ & $227 \%$ & 0.42 \\
\hline \multirow[t]{2}{*}{$(9)+(17)$} & Complete EIT image & $0.735 \pm 0.210$ & $-0.004 \pm 0.236$ & $32 \%$ & 0.96 \\
\hline & Pneumotachometer $\mathrm{b}^{\mathrm{b}}$ & $0.749 \pm 0.244$ & & & \\
\hline
\end{tabular}

a Calibration equations for COPD patients. These were obtained by adding the equations for healthy males (see Table 1 ) and the adjusted equations obtained in the group of COPD patients (see Table 3).

b The pneumotachometer is considered in this study as gold standard.

c This value is the volume obtained by pneumotachometer.

d Volume determined by using the adjusted calibration equation (i) for COPD patients.

e The volume dispersion was determined respect to the mean of volume measurements obtained by pneumotachometer.

fE32p and E25p: elliptical areas with major axis of 32 and 25 pixels, respectively; M70 and M30: ROI's obtained by mouse method; P70 and P30: ROI's obtained by percentage method.

$\mathrm{g}$ The mean estimated volume and the mean volume differences are expressed as mean \pm standard deviation.

\subsection{Results in COPD patients}

The anthropometric values of the obtaining subgroup COPD:16 were as follows: age: $63 \pm 9$ years, height: $1.65 \pm 0.05$ $\mathrm{m}$, weight: $76 \pm 11 \mathrm{~kg}, \mathrm{BMI}: 28 \pm 4 \mathrm{~kg} / \mathrm{m} 2$. The thoracic skinfold measurements at the front, at side, back and subscapular were $25 \pm 7 \mathrm{~mm}, 24 \pm 8 \mathrm{~mm}, 24 \pm 9 \mathrm{~mm}$ and $27 \pm 9 \mathrm{~mm}$, respectively. The chest circumference at rest and at maximum inspiration and expiration were $100 \pm 6 \mathrm{~cm}, 102 \pm 6 \mathrm{~cm}$ and $99 \pm 7 \mathrm{~cm}$, respectively. 
The general mathematical model obtained in this subgroup of patients that allows adjusting the calibration equation for healthy males, is determined by the following expression:

AAdjusted $=\mathrm{Cte}+\beta 1 \times \mathrm{SS} \mathrm{Sk}+\beta 2 \times$ Height $+\beta 3 \times$ Weight $+\beta 4 \times$ Frt Sk $+\beta 5 \times$ Age

where AAdjusted is the adjustment factor used to correct the calibration equations for healthy males (see Table 1); Frt Sk is the front skinfold and $\beta \mathrm{i}$ are constant coefficients. All coefficients for each equation, determined by each ROI, are shown in Table 3. Therefore, the calibration mathematical model for COPD patients is determined by the addition of the calibration equation for healthy males (Eq. (2)) and the adjustment equation for patients (Eq. (10)). So, the adjusted calibration equation for COPD patients is defined by the following expression:

ACOPD $=$ ACalibration + AAdjusted

Table 5. Test of Levene and t-test for dependent data for volume differences between the volume obtained by TIE4sys (using all EIT frame) and that obtained by TIE4sys, using all ROls. Analysis performed for healthy males and COPD patients groups.

\begin{tabular}{|c|c|c|c|c|}
\hline \multirow[t]{2}{*}{ Differences } & \multicolumn{2}{|l|}{ Healthy males } & \multicolumn{2}{|l|}{ COPD patients } \\
\hline & Levene's test ( $p$-value) & $t$-Test ( $p$-value) & Levene's test ( $p$-value) & $t$-Test ( $p$-value) \\
\hline DifVEIT100\% - DifVROIE32 & 0.993 & 0.175 & 0.503 & 0.928 \\
\hline DifVEIT100\% - DifVROIE25 & 0.781 & 0.666 & 0.532 & 0.797 \\
\hline DifVEIT100\% - DifVROIE70 & 0.456 & 0.414 & 0.863 & 0.791 \\
\hline DifVEIT100\% - DifVROIE30 & 0.042 & 0.305 & 0.080 & 0.400 \\
\hline DifVEIT100\% - DifVROIE70 & 0.577 & 0.551 & 0.180 & 0.778 \\
\hline DifVEIT100\% - DifVROIE30 & 0.091 & 0.356 & 0.089 & 0.407 \\
\hline
\end{tabular}

DifVEIT100\%: volume difference obtained by pneumotachometer and TIE4sys using all elements of EIT image.

DifVROIE32: volume difference obtained by pneumotachometer and TIE4sys using the E32 area.

DifVROIE25: volume difference obtained by pneumotachometer and TIE4sys using the E25 area.

DifVROIM70: volume difference obtained by pneumotachometer and TIE4sys using the M70 area.

DifVROIM30: volume difference obtained by pneumotachometer and TIE4sys using the M30 area.

DifVROIP70: volume difference obtained by pneumotachometer and TIE4sys using the P70 area.

DifVROIP30: volume difference obtained by pneumotachometer and TIE4sys using the P30 area.

The calibration equations for COPD patients were tested in a validation subgroup (COPD: 8). The anthropometric values of the validation subgroup were as follows: age: $71 \pm 7$ years, height: $1.65 \pm 0.10 \mathrm{~m}$, weight: $78 \pm 15 \mathrm{~kg}, \mathrm{BMI}: 29 \pm 4$ $\mathrm{kg} / \mathrm{m} 2$. The thoracic skinfold measurements at the front, at side, back and subscapular were $23 \pm 5 \mathrm{~mm}, 25 \pm 7 \mathrm{~mm}$, $25 \pm 7 \mathrm{~mm}$ and $28 \pm 6 \mathrm{~mm}$, respectively. The chest circumference at rest and at maximum inspiration and expiration were $103 \pm 8 \mathrm{~cm}, 103 \pm 7 \mathrm{~cm}$ and $101 \pm 8 \mathrm{~cm}$, respectively.

The main volume determination obtained by pneumotachometer in this group and the volumes obtained by TIE4sys, using all adjusted calibration equations determined by different ROI's, are shown in Table 4. The differences of volume measurements obtained by both monitoring devices (pneumotachometer and TIE4sys) and its respective statistical analysis (Student's t-test) are shown also in Table 4.

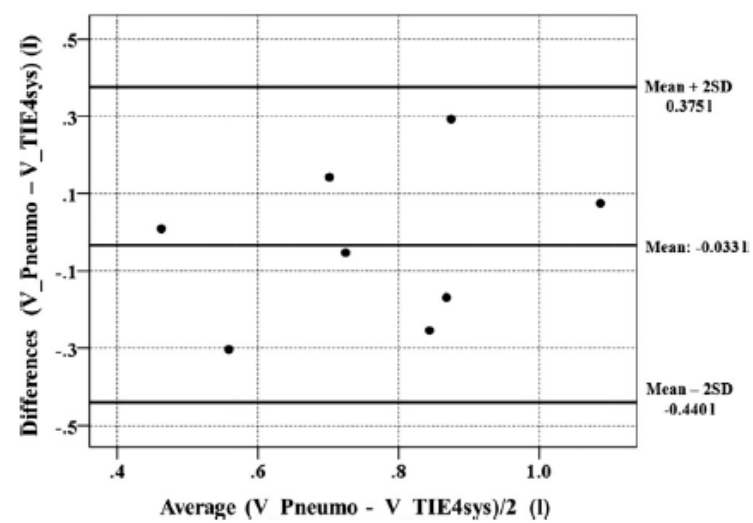

Fig. 3. Bland and Altman graphic. Dispersion of volume differences obtained by pneumotachometer and TIE4sys, using adjusted calibration model determined by E25 area and composed by Eq. (4) (see Table 1) and Eq. (12) (see Table 3).

From the statistical analysis of the results shown in Table 4, it was evidenced that the lowest volume difference was obtained by the pneumotachometer and TIE4sys by using Eq. (4) (see Table 1) and Eq. (12) (see Table 3). This mathematical model corresponds to the E25 area. However, all the volume differences analyzed are statistically consistent with zero. Also, there are not significant differences between the dispersions of volume differences obtained by the pneumotachometer and those obtained by TIE4sys using each ROI (see Table 5). 
The volume differences determined by E25 were analyzed by a Bland-Altman graphic (see Fig. 3). The mean of volume differences for this case was of $-0.033 \pm 0.204$ I with upper and lower limits of 0.375 and -0.440 I, respectively. The corresponding linear regression graphic and its respective equation of the volume measurements obtained by the pneumotachometer and EIT system, are shown in Fig. 4. In this graphic, a non-statistically significant and low value for the coefficient of determination is obtained $(R 2=0.35, p>0.05)$. This value is lower than the one obtained in the group of healthy males.

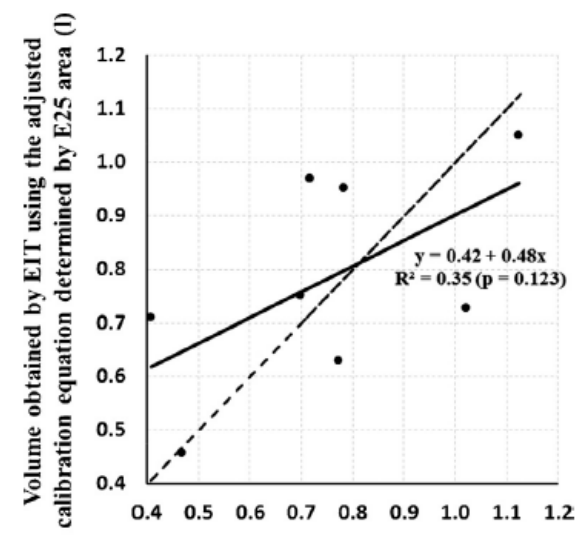

Volume obtained by pneumotachometer (l)

Fig. 4. Linear regression graphic of volume determinations obtained by pneumotachometer and TIE4sys system, using the adjusted calibration equation determined by E25 area and composed by Eq. (4) (see Table 1) and Eq. (12) (see Table 3). Dashed line represents identical results for both techniques.

\section{Discussion}

In previous works, a set of calibration equations for healthy volunteers and COPD patients were obtained. All of them were determined by a multivariate linear regression analysis of anthropometric parameters, corresponding to each group, and the proportionally coefficients. This latter parameter is determined by the quotient of impedance and volume determinations obtained by TIE4sys and pneumotachometer (used as gold standard), respectively.

From the obtained results, it was evidenced that the mathematical adjustment of calibration equations in males was better than females. For the case of COPD patients was necessary to adjust the calibration equations of healthy males with a mathematical model.

The main objective of this research was to find out if it is possible to reduce or maintain the dispersion of volume differences obtained by a pneumotachometer and EIT device, using 6 different areas of interest, in a healthy male sample and in a group of COPD patients. The use of regions of interest can be justified by the fact that EIT image by itself gives relative information about respiration changes through a cross-section of the thorax. The regions proposed in this study are placed in the central area (corresponding to each lung) of the EIT images. In this way, the border zones of the lungs, which are not defined in EIT images, were avoided. Therefore, all pixels belonging to ROIs showed the greatest impedance variations, having a higher probability to belong to the lungs.

EIT imaging depends strongly on anthropometrical differences among subjects and other physiological conditions (e.g. parenchyma condition), therefore the need of subject stratification is mandatory. Therefore, this work considers only one gender and makes the difference between healthy and non-healthy (pulmonary related illness as COPD) groups. For the case of healthy males, the most significant parameters involved in the calibration equations, for 4 of the 6 ROI's and the whole EIT image, were the subscapular skinfold and weight. The common parameter of the remainder equations was the body mass index (see Table 1). The mean value of determination coefficients (R2) of all mathematical calibration models was $0.94 \pm 0.02$. Subsequently, all equations were assessed in a validation group $(\mathrm{H}: 8)$. The lowest volume differences obtained by pneumotachometer and EIT device were determined by the areas M70 (Eq. (5)) and P70 (Eq. (7)), see Table 1. Both results showed an average volume dispersion of $9 \%$ that represent a $25 \%$ improvement with respect to the $12 \%$ volume dispersion in average from EIT results. This value depicts an improvement of $3 \%$ with respect to the result determined by the whole EIT image (see Table 2). The regions M30 and P30 seem to be a bad option to monitor pulmonary ventilation. With the first (M30) mask, it was obtained a volume dispersion of $407 \%$ (see Table 2). This large dispersion was mainly due to the results obtained in two volunteers. However, independently of these particular cases, we think that this area could be too small to give an average of ventilation information of whole lung. The same effect was observed with the use of the second mask (P30), now with the lack of adjustment of the results corresponding to one volunteer. Therefore, we discarded these two options from patient's analysis. For the case of COPD patients, it was necessary to adjust each calibration model of healthy males by applying its corresponding adjustment equation obtained in the group of COPD patients (COPD:16). This adjustment (Eq. (18) and Table 3) characterizes the condition of sickness, in our case the COPD, in a group of subjects. The most important parameters involved in the COPD adjustment equations were subscapular skinfold and height. 
The mean value of determination coefficients (R2) of these mathematical models was $0.90 \pm 0.02$. The lowest average volume differences obtained by pneumotachometer and EIT device were determined by the area E25 (Eq. (4) + Eq. (12), see Table 4) with a value of $27 \%$ (see Table 4). This value represents an improvement of $15.6 \%$ with respect to the $32 \%$ volume dispersion in average obtained using whole image EIT technique. Considering that the pneumotachometer by itself produce respiratory pattern alteration around $15-35 \%$ as it was mentioned in the introduction, and that the volume difference dispersions are around $9 \%$ (for healthy group) to $27 \%$ (for COPD group) using the ROI's, it is possible to suggest that the use of ROI's does not affect negatively the agreement of the volume estimation compared with the complete EIT image technique and even compared with the gold standard. This is stated for the volume range of the results for each group (see Fig. 1 for the case of healthy males and Fig. 3 for the case of COPD patients).

All these results were obtained within a small range of tidal volumes (0.4-0.9), therefore the conclusions should be restricted to this volume range. Also the huge volume dispersion in COPD group suggests the need of further stratification according to the particular pathology or parenchyma condition.

\section{Conclusions}

For the case of healthy males, the lowest volume differences were evidenced by using the M70 and P70 area. Nevertheless, all the volume differences analyzed are statistically consistent with zero and the dispersions of volume differences (variance) determined by all regions of interest, including the use of all elements of EIT image, do not show significant differences between them, excepting the M30 area. For the case of COPD patients, all the volume differences analyzed are again statistically consistent with zero. In this case the dispersions of volume differences (variance) are larger than those obtained for healthy subjects, but again those dispersions, determined by all areas of interest, do not show significant differences between them. These results could suggest the need for a stratification taking into account the parameters describing the physiological condition of the lung parenchyma. Nevertheless, for monitoring purpose, the use of ROI's could be useful also in this group.

Summarizing ROI's alternative gives similar results to the use of all elements of EIT image with similar dispersions of the volume determinations to those reported for the gold standard, considering the volume range limitations. For COPD subjects further investigations are needed to improve volume estimation.

\section{Acknowledgments}

Dirección de Investigación y Posgrado (DAIP) (FO-DAI-05), Universidad de Guanajuato for financial support and PROMEP-SEP (postdoctoral fellowship) to Marco Balleza.

\section{References}

[1] J. Sanchis, L. Díez-Betoret, J. Canet, P. Casan, Pneumotachographic recording of ventilatory pattern, in: S. Benito, A. Net (Eds.), Update in Intensive Care and Emergency Medicine 13: Pulmonary Function in Mechanically Ventilated Patients, SpringerVerlag, Berlin-Heidelberg, 1991, pp. 9-22.

[2] X. Serra Gabriel, Anatomía básica del aparato respiratorio, En: María Rosa Serra Gabriel, Josefina Díaz Petit y María Luisa de Sande Carril (Comp), Fisioterapia en neumología, sistema respiratorio y aparato cardiovascular, Elsevier, Barcelona, 2005, pp. 281-293.

[3] R. Gilbert, J.H. Auchincloss, J. Brodsky, W. Boden, Changes in tidal volume, frequency and ventilation induced by their measurement, J. Appl. Physiol. 33 (1972) 252-254.

[4] J. Askanazi, P.A. Silverberg, R.J. Foster, A.I. Hyman, Effects of respiratory apparatous on breathing pattern, J. Appl. Physiol. 48 (1980) 577-580.

[5] W. Perez, M.J. Tobin, Separation of factor responsible for change in breathing pattern induced by instrumentation, J. Appl. Physiol. 59 (1985) 1515-1520.

[6] D. Paek, D. McCool, Breathing patterns during varied activities, J. Appl. Physiol. 73 (1992) 887-893.

[7] K.F. Whyte, M. Gugger, G.A. Gould, J. Molloy, P.K. Wraith, N.J. Douglas, Accuracy of respiratory inductive plethysmograph in measuring tidal volume during sleep, J. Appl. Physiol. 71 (1991) 1866-1871.

[8] S.M. Stick, E. Ellis, P.N. LeSouëf, P.D. Sly, Validation of respiratory inductance plethysmography ("Respitrace") for the measurement of tidal breathing parameters in newborns, Pediatr. Pulmonol. 14 (3) (1992) 187-191.

[9] K.P. Cohen, W.M. Ladd, D.M. Beams, W.S. Sheers, R.G. Radwin, W.J. Tompkins, J.G. Webster, Comparison of impedance and inductance ventilation sensors on adults during breathing, motion and simulated airway obstruction, IEEE Trans. Biomed. Eng. 44 (7) (1997) 555-566

[10] B.H. Brown, A.D. Seagar, The Sheffield data collection system, Clin. Phys. Physiol. Meas. 8 (Suppl. A) (1987) 91-97.

[11] B.H. Brown, Electrical impedance tomography (EIT): a review, J. Med. Eng. Technol. 27 (2003) 97-108.

[12] M. Bodenstein, M. David, K. Markstaller, Principles of electrical impedance tomography and its clinical application, Crit. Care Med. 37 (2) (2009) 713-724.

[13] S. Leonhardt, B. Lachmann, Electrical Impedance Tomography: the holy grail of ventilation and perfusion monitoring? Intensive Care Med. 38 (2012) 1917-1929.

[14] E.L. Costa, C.N. Chaves, S. Gomes, M.A. Beraldo, M.S. Volpe, M.R. Tucci, I.A. Schettino, S.H. Bohm, C.R. Carvalho, H. Tanaka, R.G. Lima, M.B. Amato, Real-time detection of pneumothorax using electrical impedance tomography, Crit. Care Med. 35 (2008) 1230-1238. 
[15] C. Preis, H. Luepschen, S. Leonhardt, D. Gommers, Experimental case report: development of a pneumothorax monitored bye electrical impedance tomography, Clin. Physiol. Funct. Imaging 29 (2009) 159-162.

[16] P.W. Kunst, A. Vonk Noordegraaf, E. Raaijmakers, J. Bakker, A.B. Groeneveld, P.E. Postmus, P.M. de Vries, Electrical impedance tomography in the assessment of extravascular lung water in noncardiogenic acute respiratory failure, Chest 116 (1999) 1695-1702.

[17] M. Arad, S. Zlochiver, T. Davidson, Y. Shoenfeld, A. Adunsky, S. Abboud, The detection of pleural effusion using parametric EIT technique, Physiol. Meas. 30 (2009) 421-428.

[18] P.W. Kunst, S.H. Bohm, G. Vazquez de Anda, M.B. Amato, B. Lachmann, P.E. Postmus, P.M. de Vries, Regional pressure volume curves by electrical impedance tomography in a model of acute lung injury, Crit. Care Med. 28 (2000) 178-183.

[19] B. Grychtol, G.K. Wolf, J.H. Arnold, Differences in regional pulmonary pressure impedance curves before and after lung injury assessed with a novel algorithm, Physiol. Meas. 30 (2009) 137-148.

[20] I. Frerichs, P.A. Dargaville, P.C. Rimensberger, Regional respiratory inflation and deflation pressure-volume curves determined by electrical impedance tomography, Physiol. Meas. 34 (2013) 567-577.

[21] T. Bein, F. Ploner, M. Ritzka, M. Pfeifer, H.J. Schlitt, B.M. Graf, No change in the regional distribution of tidal volume during lateral posture in mechanically ventilated patients assessed by electrical impedance tomography, Clin. Physiol. Funct. Imaging 30 (2010) 234-240.

[22] P.A. Dargaville, P.C. Rimensberger, I. Frerichs, Regional tidal ventilation and compliance during a stepwise vital capacity manoeuvre, Intensive Care Med. 36 (2010) 1953-1961.

[23] F. Reifferscheid, G. Elke, S. Pulletz, B. Gawelczyk, I. Lautenschläger, M. Steinfath, N. Weiler, I. Frerichs, Regional ventilation distribution determined by electrical impedance tomography: reproducibility and effects of posture and chest plane, Respirology 16 (2011) 523-531.

[24] C. Gómez-Laberge, J.H. Arnold, G.K. Wolf, A unified approach for EIT imaging of regional overdistension and atelectasis in acute lung injury, IEEE Trans. Med. Imaging 31 (3) (2012) 834-842.

[25] M. Czaplik, I. Biener, R. Dembinski, P. Pelosi, T. Soodt, W. Schroeder, S. Leonhardt, G. Marx, R. Rossaint, J. Bickenbach Analysis of regional compliance in a porcine model of acute lung injury, Respir. Physiol. Neurobiol. 184 (2012) 16-26.

[26] T. Muders, H. Luepschen, J. Zinserling, S. Greschus, R. Fimmers, U. Guenther, M. Buchwald, D. Grigutsch, S. Leonhardt, C. Putensen, H. Wrigge, Tidal recruitment assessed by electrical impedance tomography and computed tomography in a porcine model of lung injury, Crit. Care Med. 40 (3) (2012) 903-911.

[27] G. Elke, M.K. Fuld, A.F. Halaweish, B. Grychtol, N. Weiler, E.A. Hoffman, I. Frerichs, Quantification of ventilation distribution in regional lung injury by electrical impedance tomography and xenon computed tomography, Physiol. Meas. 34 (2013) 13031318.

[28] G.K. Wolf, C. Gómez-Laberge, J.S. Rettig, S.O. Vargas, C.D. Smallwood, S.P. Prabhu, S.H. Vitali, D. Zurakowski, J.H. Arnold, Mechanical ventilation guided by electrical impedance tomography in experimental acute lung injury, Crit. Care Med. 41 (5) (2013) 1296-1304

[29] M. Balleza, J. Fornos, T. Feixas, M. González, D. Antón, P.J. Riu, P. Casan, Monitoring of breathing pattern at rest by electrical impedance tomography, Arch. Bronconeumol. 43 (2007) 300-303.

[30] M. Balleza, N. Calaf, T. Feixas, M. González, D. Antón, P.J. Riu, P. Casan, Measuring breathing pattern in patients with chronic obstructive pulmonary disease by electrical impedance tomography, Arch. Bronconeumol. 45 (2009) 320-324.

[31] S. Nebuya, G.H. Mills, P. Milnes, B.H. Brown, Indirect measurement of lung density and air volume from electrical impedance tomography (EIT) data, Physiol. Meas. 32 (2011) 1953-1967.

[32] B. De Lema, P. Casan, P.J. Riu, Electrical Impedance Tomography: standardizing in the procedure in pneumology, Arch. Bronconeumol. 42 (2006) 299-301.

[33] O. Casas, Contribución a la obtención de imágenes paramétricas en tomografía de impedancia eléctrica para la caracterización de tejidos biológicos (Tesis Doctoral), UniversitatPolitècnica de Catalunya, Barcelona, 1998.

[34] A. Fontova, Desenvolupament d'un mòdul de comunicacions Ethernet per a un sistema de TIE. Projecte fi de Carrera. E.T.S.E.T.B. UniversitatPolitècnica de Catalunya, 2004.

[35] R.E. Serrano, B. De Lema, O. Casas, T. Feixas, N. Calaf, V. Camacho, I. Carrio, P. Casan, J.P. Riu, Use of electrical impedance tomography (TIE) for the assessment of unilateral pulmonary function, Physiol. Meas. 23 (2002) 211-220.

[36] J. Fornos Herrando, Estimació del Patró Ventilatorimitjanc, ant Tomografía d'ImpedànciaElèctrica. Projecte fi de Carrera. E.T.S.E.T.B. Universitat Politècnica de Catalunya, 2006.

[37] T. Meier, H. Luepschen, J. Karsten, T. Leibecke, M. Großherr, H. Gehring, S. Leonhardt, Assessment of regional lung recruitment and derecruitment during a PEEP trial based on electrical impedance tomography, Intensive Care Med. 34 (3) (2008) 543-550.

[38] L. Fabbri, J.C. Hogg, C. Jenkins, E. Nizankowska-Mogilnicka, S. Sullivan, T. van der Molen, C. van Weel, Guideline: Global Strategy for the Diagnosis, Management, and Prevention of Chronic Obstructive Pulmonary Disease, Global Initiative for Chronic Obstructive Pulmonary Disease, Sociedad, 2007.

[39] P.P. Rickham, Human experimentation. Code of ethics of the world medical association. Declaration of Helsinki, Br Med J. 2 (1964) 177 (PMC free article) (PubMed).

[40] J.M. Bland, D.G. Altman, Measuring agreement in method comparison studies, Stat. Methods Med. Res. 8 (1999) $136-160$.

[41] J.M. Bland, D.G. Altman, Statistical methods for assessing agreement between two methods of clinical measurement, Lancet 1 (1986) 307-310 